\title{
Perfil de pacientes con enfermedades minoritarias en Galicia. Perspectiva desde la medicina interna
}

\author{
Clinical profile of patients with rare diseases. \\ An approach from Internal Medicine
}

\begin{abstract}
Alberto Rivera Gallego ${ }^{1}$, Susana Rivera García ${ }^{2}$, Ana Arévalo Gómez², Beatriz Buño Ramilo³ ${ }^{3}$ Ana María Bravo Blanco ${ }^{4}$, Roi Suárez Gil ${ }^{5}$, Enrique Chao Villar ${ }^{6}$, Álvaro Hermida-Ameijeiras ${ }^{6}$

${ }^{1}$ Servicio de Medicina Interna. Xerencia Xestión Integrada área de Vigo. ${ }^{2}$ Servicio de Medicina Interna. Xerencia Xestión Integrada área de A Coruña y Cee. ${ }^{3}$ Servicio de Medicina Interna. Xerencia Xestión Integrada área de Ferrol. ${ }^{4}$ Servicio de Medicina Interna. Xerencia Xestión Integrada área de Ourense, Verín y 0 Barco de Valdeorras. ${ }^{5}$ Servicio de Medicina Interna. Xerencia Xestión Integrada área de Lugo, A Mariña y Monforte de Lemos. ${ }^{6}$ CSUR Enfermedades Metabólicas Hereditarias de Santiago de Compostela. Servicio de Medicina Interna. Xerencia Xestión Integrada área de Santiago de Compostela y Barbanza. Área de Medicina. Universidade de Santiago de Compostela.
\end{abstract}

\section{RESUMEN}

Objetivos: Las enfermedades minoritarias constituyen un grupo heterogéneo de patologías de baja prevalencia, con un origen genético en la mayoría de los casos y frecuentemente asociadas a un retraso en su diagnóstico y notable morbi-mortalidad. Conocer las características clínicas y el grado de complejidad asistencial de los pacientes con enfermedades minoritarias que acuden a las consultas de medicina interna en Galicia podría facilitar una atención más eficaz y eficiente.

Material y métodos: Estudio epidemiológico, transversal y multicéntrico en pacientes con enfermedad de baja prevalencia $\geq 18$ años atendidos en las consultas y unidades específicas de medicina interna en Galicia hasta el 31 de Diciembre de 2020. Se obtuvieron de cada centro el número de pacientes atendidos según la patología, la media en años desde la aparición de los síntomas, número de comorbilidades, número de visitas anuales al centro y a otros especialistas, número de tratamientos (incluidos aquellos de alto impacto económico), grado de deterioro cognitivo y estimación del nivel de dependencia.

Resultados: Se analizaron los indicadores de seis centros correspondientes a seis áreas sanitarias de Galicia, representando a un total de 324 pacientes, con una edad media de 41,3 $\pm 15,8$ años. Los tres principales grupos de patologías atendidas fueron por este orden las enfermedades genéticas raras, los errores innatos del metabolismo y las enfermedades neurológicas raras. El retraso medio en el diagnóstico fue de 4,8 $\pm 7,9$ años y un $34,17 \%$ de los pacientes tardaron 5 o más años en tener una confirmación diagnóstica. Este grupo de mayor retraso diagnóstico presenta menor puntuación en la escala de dependencia de Barthel y 1,75 veces mayor utilización de recursos sanitarios (consultas a medicina interna y otras especialidades). El $11,75 \%$ de los pacientes presentan un nivel de dependencia severa o total y el $9,9 \%$, un bajo coeficiente intelectual.

Conclusiones: Los pacientes con enfermedades minoritarias atendidos en los servicios de medicina interna en Galicia presentan a pesar de su baja media de edad, una elevada complejidad asistencial derivada del número de comorbilidades, asistencias a consultas, la necesidad de ingresos hospitalarios y la polifarmacia. Complejidad que guarda relación estrecha con el retraso en el diagnóstico.

Palabras Clave: Enfermedades raras, enfermedades minoritarias, retraso diagnóstico, gestión de la atención, índice de Barthel, comorbilidades.

\section{INTRODUCCIÓN}

Bajo la denominación de enfermedades minoritarias, huérfanas o raras (EEMM), se agrupan más de 7000 entidades nosológicas con una gran heterogeneidad clínica que comparten algunos aspectos en común. Todas ellas tienen una prevalencia inferior a un caso por cada 2.000 habitantes (el equivalente a un $7 \%$ de la población mundial afectada y

\section{ABSTRACT}

Objectives: Rare diseases $(\mathrm{RD})$ constitute a heterogeneous group of low prevalence conditions, with genetic origin in most cases and frequently associated with a delay in their diagnosis and notable morbidity and mortality. Knowing the clinical profile and the complexity degree of patients with RD who attend internal medicine units in Galicia could facilitate more effective and efficient care settings.

Methods: Epidemiological, cross-sectional and multicenter study in patients with low prevalence diseases $\geq 18$ years attending the outpatients departments and specific units of internal medicine in Galicia up to December 31, 2020. Data were collected on the number of patients treated regarding their condition at each center, the average age at disease onset, number of comorbidities, number of annual visits to the center and other specialists, number of treatments (including those with a high economic impact), degree of cognitive impairment and assessment of the degree of autonomy.

Results: We analyzed data from six participating centers (from six health areas of Galicia), representing a total of 324 patients, with a mean age of $41.3 \pm 15.8$ years. The three main groups of pathologies treated were, in this order, rare genetic diseases, innate errors of metabolism and rare neurological diseases. The mean delay in diagnosis was $4.8 \pm 7.9$ years and $34.17 \%$ of patients took 5 or more years to have a diagnostic confirmation. This group with the longest diagnostic delay has a lower score on the Barthel dependency scale and 1.75 times greater use of health resources (consultations with internal medicine and other specialties). $11.75 \%$ of the patients present a level of severe or total dependence and 9.9\%, a low IQ.

Conclusions: Despite their low average age, patients with minority diseases treated in internal medicine services present a high complexity of care derived from the number of comorbidities, visits to consultations, the need for hospital admissions and polypharmacy. Complexity that is closely related to the delay in diagnosis.

Keywords: Rare Disease, Minority Diseases, diagnosis delay, healthcare management, Barthel Index, comorbidities

alrededor de 3 millones de españoles, 200.000 en la comunidad gallega) ${ }^{1,2}$. En más del $80 \%$ de los casos, el origen es genético y en muchas ocasiones se asocian a una evolución crónica severa, provocando múltiples deficiencias motoras, sensoriales y cognitivas así como elevada morbimortalidad.

Es precisamente la baja prevalencia sumada a la falta de conocimientos médico-científicos sobre estas enfermedades 
lo que ocasiona una importante demora en su diagnóstico y su abordaje asistencial, estimado en un tiempo medio de 5 a 10 años ${ }^{2,3}$. Período este en el que los pacientes se ven obligados a frecuentar numerosas especialidades médicas y se acompaña de un elevado coste económico y emocional para el paciente y la familia ${ }^{4}$. Por otro lado, también la baja prevalencia de estas enfermedades es una de las principales causas que limita las opciones terapéuticas junto con la falta de inversión y el elevado coste de los medicamentos "huérfanos" (término acuñado para designar al medicamento destinado a la prevención, diagnóstico o tratamiento de las EEMM) $)^{5}$. Es importante destacar que los pacientes con EEMM presentan habitualmente numerosas comorbilidades para los que reciben medicamentos convencionales y que contribuye a incrementar el gasto farmacéutico de cada familia (que puede alcanzar de media los $350 €$ mensuales, un $25 \%$ de los ingresos familiares) sin que existan, muchas veces, políticas sociales que amparen este impacto socio-familiar, ${ }^{6,7}$.

Uno de los principales retos en la atención clínica a los pacientes con EEMM es la existencia de estructuras asistenciales coordinadas y especializadas capaces de garantizar la continuidad de la atención sanitaria de la edad pediátrica a la adulta, estabilizar el curso clínico de la enfermedad y al mismo tempo detectar cualquier cambio que sugiera la progresión o descompensación de la misma. Con este fin se promueve la creación de equipos multidisciplinares en los que el médico internista se ha convertido en el eje central para coordinar la atención al paciente adulto con EEMM². Tal y como se recoge en el programa formativo de la especialidad (Orden SCO/227/2007 de 24 de enero), la atención al paciente con EEMM es uno de los campos de acción del especialista en formación de medicina interna y por tanto no solo ha de trabajar en equipo en colaboración con otros especialistas hospitalarios y con el médico de familia sino que puede participar activamente en labores asistenciales e investigadoras ${ }^{5,8}$.

El objetivo principal del presente estudio es analizar el perfil y grado de complejidad asistencial de los pacientes adultos con EEMM atendidos de forma regular por los servicios de medicina interna en las consultas específicas, unidades y centros de referencia para Enfermedades Minoritarias en Galicia.

\section{MATERIAL Y MÉTODOS}

Se realizó un estudio observacional y retrospectivo a través del Grupo de trabajo en Enfermedades Minoritarias de la Sociedad Gallega de Medicina Interna, en el que se recogieron distintos indicadores asistenciales de las consultas y unidades específicas en los principales centros hospitalarios de Galicia hasta el 31 de Diciembre de 2020.

Los datos recogidos representan a una población de estudio de 324 pacientes atendidos en las consultas monográficas de medicina interna en las Gerencias de Gestión Integrada de A Coruña, Ferrol, Lugo, Santiago, Ourense y Vigo.
Se recogieron de cada unidad o consulta, los siguientes indicadores asistenciales; número de pacientes atendidos, distribución por patología, edad, sexo, media en años en el retraso diagnóstico (desde la aparición de los síntomas), número de comorbilidades, número de visitas a otros especialistas, número de tratamientos (incluidos aquellos de "alto impacto económico"), número de familiares afectados por la misma condición y grado de deterioro cognitivo de los pacientes en base al coeficiente intelectual (calculado mediante la escala Wechsler).

Si bien no existen clasificaciones validadas para las EEMM, se utilizó para las diferentes entidades recogidas en nuestro estudio, una clasificación jerárquica ampliamente extendida que establece ORPHANET (consorcio europeo coordinado por el Institut National de la Santé et de la Recherche Médicale, INSERM) y que permite tener una visión de conjunto según las dolencias que provocan?.

En aquellos casos en los que estaba disponible, se recogieron los valores de funcionalidad en la realización de las actividades de la vida diaria, estimados mediante el Índice de Barthel (BB), asignando una puntuación total de 100 para la máxima independencia y de 0 la de máxima dependencia. Se consideró como paciente autónomo aquel que obtuvo 100 puntos de 100, dependiente leve aquel que obtuvo un puntaje mayor a 60, dependiente severo aquel que puntúa entre más de 30 y menos de 60 y dependiente total, aquel que registra menos de 30 puntos $^{10}$.

Se realizó un estudio descriptivo de las variables incluidas en el estudio. Las variables cualitativas fueron expresadas como valor absoluto y porcentaje. Las variables cuantitativas se expresaron como media y su correspondiente desviación estándar. En el análisis estadístico se utilizó la técnica Chi cuadrado con la corrección de Yates cuando fue preciso, en la comparación de las variables cualitativas. En la comparación de dos variables cuantitativas se utilizó el test T de Student, previa comprobación de normalidad. El test de ANOVA se empleó para el análisis de variables con más de 2 categorías (nivel de dependencia). Se ajustó un modelo de regresión logística explicativo con el objetivo de estimar el efecto que el nivel de dependencia tiene en relación con los diferentes índices de complejidad asistencial. El nivel de significación estadística fue $p<0,05$. En el análisis se utilizó el paquete estadístico SPSS 25.

\section{RESULTADOS}

La media de edad de los pacientes atendidos se sitúa en 41,3 $\pm 15,8$ años) y un $60,4 \%$ de mujeres. Las principales EEMM atendidas en los servicios de medicina interna de Galicia a través de sus consultas y unidades específicas son la Fenilcetonuria (n: 40); la enfermedad de Fabry (n: 21), la enfermedad de Rendu-Osler-Weber (n: 20), la Esclerosis Tuberosa (n: 16) y la enfermedad de Gaucher (n: 10). La distribución de las diferentes entidades en base a la clasificación para las EEMM que establece ORPHANET, se representa en la Tabla 1. 
Tabla 1. Distribución de las EEMM atendidas en las Unidades y consultas monográficas de medicina interna de acuerdo a la clasificación establecida por ORPHANET9.

\begin{tabular}{|l|c|c|}
\hline Enfermedades genéticas raras & 245 & $75,6 \%$ \\
\hline Errores innatos del metabolismo & 161 & $49,7 \%$ \\
\hline Enfermedades neurológicas raras & 139 & $42,9 \%$ \\
\hline $\begin{array}{l}\text { Anomalías raras del desarrollo durante la } \\
\text { embriogénesis }\end{array}$ & 93 & $28,7 \%$ \\
\hline Enfermedades dermatológicas raras & 89 & $27,5 \%$ \\
\hline Enfermedades renales raras & 86 & $26,5 \%$ \\
\hline Enfermedades raras del sistema circulatorio & 71 & $21,9 \%$ \\
\hline Enfermedades raras oftalmológicas & 66 & $20,4 \%$ \\
\hline Enfermedades raras hepáticas & 44 & $13,6 \%$ \\
\hline Enfermedades raras cardíacas & 33 & $10,2 \%$ \\
\hline Enfermedades neoplásicas raras & 33 & $10,2 \%$ \\
\hline Enfermedades raras respiratorias & 28 & $8,6 \%$ \\
\hline Enfermedades raras del sistema óseo & 25 & $7,7 \%$ \\
\hline Enfermedades raras sistémicas y reumatológicas & 17 & $5,2 \%$ \\
\hline Enfermedades gastrointestinales raras & 11 & $3,4 \%$ \\
\hline Enfermedades raras otorrinolaringológicas & 5 & $1,5 \%$ \\
\hline Trastornos por efectos tóxicos raros & 5 & $1,5 \%$ \\
\hline Enfermedades endocrinas raras & 5 & $1,5 \%$ \\
\hline Trastornos teratológicos raros & 5 & $1,5 \%$ \\
\hline $\begin{array}{l}\text { Enfermedades raras que requieren cirugia } \\
\text { abdominal }\end{array}$ & 3 & $1,2 \%$ \\
\hline $\begin{array}{l}\text { Enfermedades raras que requieren cirugia } \\
\text { torácica }\end{array}$ & $0,9 \%$ \\
\hline $\begin{array}{l}\text { Enfermedades raras que requieren cirugía } \\
\text { maxilofacial }\end{array}$ & $0,6 \%$ \\
\hline Trastornos raros que inducen infertilidad & $3 \%$ \\
\hline Trastornos raros ginecológicos y obstétricos & 3 \\
\hline
\end{tabular}

Las enfermedades están recogidas en tantas clasificaciones como sean necesarias para su descripción, dependiendo de su naturaleza.

Se estima un retraso medio en el diagnóstico de 4,8 47,9 años y destaca el hecho de que un $34,17 \%$ de los pacientes tardaron 5 o más años en ser diagnosticados desde la aparición de sus síntomas. El principal punto de derivación de los pacientes procede por este orden; de los servicios pediátricos, atención primaria, medicina interna y estudios genéticos familiares.

Respecto a los diversos índices de complejidad asistencial en la muestra a estudio, cabe resaltar pese a la corta edad media de los pacientes atendidos, el elevado número de comorbilidades (2,6 de media), con un $31,5 \%$ de pacientes que presentaron 30 más comorbilidades. Del mismo modo, un total de 80 pacientes $(24,6 \%)$ acudieron a más de 3 consultas con otras especialidades médico-quirúrgicas en el último año y 65 pacientes (20\%) precisaron 3 o más consultas en el propio servicio de medicina interna. En el último año, un 12,4\% de la población precisó ingreso hospitalario por causa de su proceso de base al menos una vez, y 20 pacientes precisaron ingresar 20 más veces $(6,2 \%)$.

El número medio de fármacos por paciente es de 2,5 (excluyendo cualquier tipo de suplemento alimentario) y 53 $(16,4 \%)$ de nuestros pacientes reciben 50 más fármacos diarios, de los cuales 28 de ellos reciben tratamientos de alto impacto económico (8,65\% de los pacientes atendidos). Un total de 32 pacientes presenta deterioro del nivel intelectual estimado mediantela escala Wechsler $(\mathrm{Cl}<80)$, lo que equivale a un $9,9 \%$ de la población a estudio. La estratificación de la muestra en base al grado de dependencia (estimado mediante el índice de Barthel) se recoge en el gráfico 1.

En la tabla 2 se representa el análisis univariado de las variables clínicas e índices de complejidad asistencial que se relacionan con el nivel de dependencia agrupado en 4 categorías: autónomo, dependencia leve, dependencia

Tabla 2. Análisis univariado. Relación de las variables clínicas e índices de complejidad asistencial en función del grado de dependencia.

\begin{tabular}{|l|c|c|c|c|c|}
\hline & Autónomo & Leve & Severa & Total & Sig (p) \\
\hline Edad (años) & 39,3 & 41,55 & 33,57 & 33 & 0,19 \\
\hline Edad al diagnóstico (años) & 25,62 & 28 & 28,43 & 20,13 & 0,554 \\
\hline Retraso diagnóstico (años) & 5 & 5,73 & 12,43 & 7,88 & 0,002 \\
\hline Comorbilidades (n) & 2,26 & 4,18 & 4,86 & 3,63 & 0,001 \\
\hline Especialistas (n) & 1,81 & 3,18 & 4,29 & 2,63 & 0,009 \\
\hline Consultas año (n) & 1,68 & 1,91 & 3,14 & 2 & 0,017 \\
\hline Ingresos hospitalarios año (n) & 0,46 & 0,9 & 1,43 & 0,75 & $<0,01$ \\
\hline Fármacos (n) & 2,09 & 3,64 & 4,43 & 3,63 & 0,001 \\
\hline
\end{tabular}


severa y dependencia total. Las variables que presentaron diferencias estadísticamente significativas entre las categorías de estudio fueron el retraso en el diagnóstico, el número de comorbilidades, número de consultas al año, número de ingresos hospitalarios en el último año, número de fármacos por cada paciente y el número de especialistas al que acude cada paciente. No se encontraron diferencias significativas en cuanto a la edad de los pacientes o el momento del diagnóstico.

En el análisis multivariante los individuos con un retraso en el diagnóstico igual o mayor a 5 años presentaron 1,75 veces más de riesgo de realizar una mayor utilización de recursos.

La tasa de prevalencia en el número de comorbilidades, ingresos hospitalarios en el último año y número de fármacos fue superior entre aquellos pacientes en nuestra muestra que aglutinan un mayor retraso diagnóstico (5 años 0 superior) con respecto a los que se diagnosticaron antes, sin alcanzar significación estadística. Sin embargo, la edad al diagnóstico, el número de consultas ambulatorias anuales así como el número de especialidades médicas visitadas, sí resultaron significativamente superiores en el grupo con mayor retraso diagnóstico (Tabla 3).

De igual modo, los pacientes con menor puntuación en la escala de dependencia de Barthel, fueron los que presentaron mayor retraso en el diagnóstico (la media de años fue de 5; 5,$73 ; 12,43$ y 7,88 para los pacientes con plena autonomía, dependencia leve, severa o dependencia total, respectivamente; nivel de significación p: 0,02). En la figura 2 se representa la distribución porcentual comparativa del grado de dependencia en el grupo de pacientes con mayor o menor retraso diagnóstico.

\section{DISCUSIÓN}

Las EEMM se asocian a una elevada complejidad debido fundamentalmente a su baja prevalencia, su cronicidad y a su comorbilidad. Existen más de 7.000 EEMM reconocidas y aunque la afectación de cada una de ellas incluye a pocos afectados, el total de todas, supone una población de más de 200.000 personas en nuestra comunidad ${ }^{1}, 2$. En muchas ocasiones la confirmación diagnóstica llega tras un largo periplo, de paso por diferentes consultas y especialistas durante el cual va aumentando su afectación física, psicológica y social2,11. Aunque la mayoría son genéticasy están presentes al nacimiento, no semanifiestan hasta edades posteriores.

Los pacientes de nuestra muestra representan al colectivo de pacientes con EEMM atendidos en las consultas monográficas y Unidades de referencia para enfermedades de baja prevalencia en una muestra amplia de los servicios de medicina interna en nuestra Comunidad. Un $75,6 \%$ de las patologías atendidas tienen una base genética lo que coincide con los datos aportados por otros organismos ${ }^{12}$.

El retraso en alcanzar un diagnóstico definitivo conlleva consecuencias tanto más graves cuanto mayor sea el tiempo transcurrido y llegando a distintos grados de discapacidad hasta la muerte, y no podemos olvidar debido a la elevada carga genética asociada a estas patologías, que la falta de un diagnóstico impide realizar un adecuado consejo genético y por tanto incrementa la posibilidad de que nazcan más recién nacidos aquejados de la misma enfermedad ${ }^{13}$. En los pacientes de nuestra muestra, el retraso medio en el diagnóstico es de 4,8 7,9 años y más de un tercio de ellos alcanzaron una confirmación diagnóstica al cabo de 50 más años, un retraso prácticamente idéntico al reportado recientemente por otros autores $^{14}$. Un análisis por grupos de la población de nuestras consultas, refleja que el mayor retardo diagnóstico se asocia significativamente con un mayor impacto clínico (mayor grado de dependencia estimada mediante el índice de Barthel) y mayor consumo de recursos sanitarios (en particular mayor número de visitas a nuestras unidades anualmente y un mayor número de especialidades médico-quirúrgicas visitadas por año). Amplios análisis retrospectivos recientemente publicados evidencian que los pacientes con EEMM tienen un 52\% más de probabilidades de ser derivados a otro especialista que los pacientes comunes ${ }^{15}$.

Los motivos que subyacen al retraso diagnóstico en las EEMM son diversos y acumulativos. Un diagnóstico tardío puede ocurrir porque el paciente no consultó lo suficientemente temprano, o porque los síntomas son inespecíficos o poco comunes. Un retraso también puede ocurrir porque el conocimiento científico es todavía limitado, o porque todas las pruebas complementarias no arrojan ningún resultado concluyente. Pero también los sistemas de salud contribuyen

Tabla 3. Tasa de prevalencia en recursos clínicos y asistenciales utilizados en relación con el grado de retraso en el diagnóstico.

\begin{tabular}{|l|c|c|c|}
\hline Variables & $<5$ años & $\geq 5$ años & Sig (p) \\
\hline Edad al diagnóstico; años (DS) & $19,46(21,1)$ & $33,02(19,1)$ & $<0,01$ \\
\hline Especialistas; $n$ (DS) & $1,62(1,1)$ & $2,78(1,7)$ & 0,317 \\
\hline Comorbilidades; $n$ (DS) & $2,33(2,17)$ & $3,33(2,39)$ & 0,008 \\
\hline Consultas año; $n$ (DS) & $1,5(1,1)$ & $2,16(1,3)$ & 0,446 \\
\hline Ingresos hospitalarios; $n$ (DS) & $0,43(1,53)$ & $0,54(1,34)$ & 0,055 \\
\hline Fármacos; $n$ (DS) & $2,08(2,8)$ & $3,04(3,3)$ & \\
\hline
\end{tabular}


Fig. 1. Distribución porcentual de la población a estudio en función del grado de dependencia (estimado mediante puntuación obtenida en el índice de Barthel).

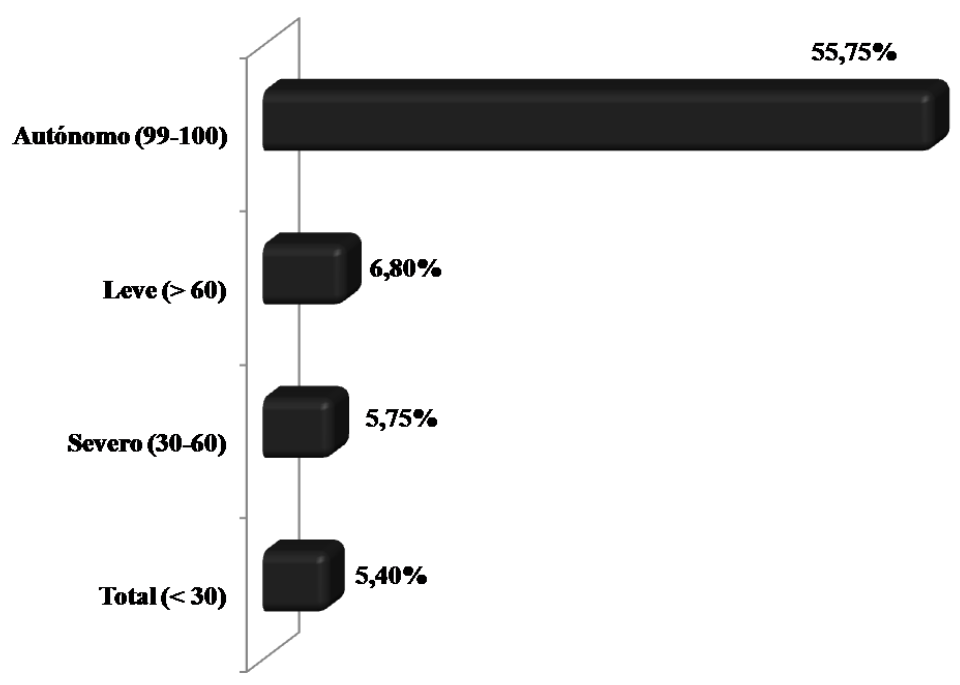

Fig. 2. Distribución porcentual comparativa del grado de dependencia (estimado mediante puntuación obtenida en el índice de Barthel) en la muestra estudiada en función del retraso diagnóstico

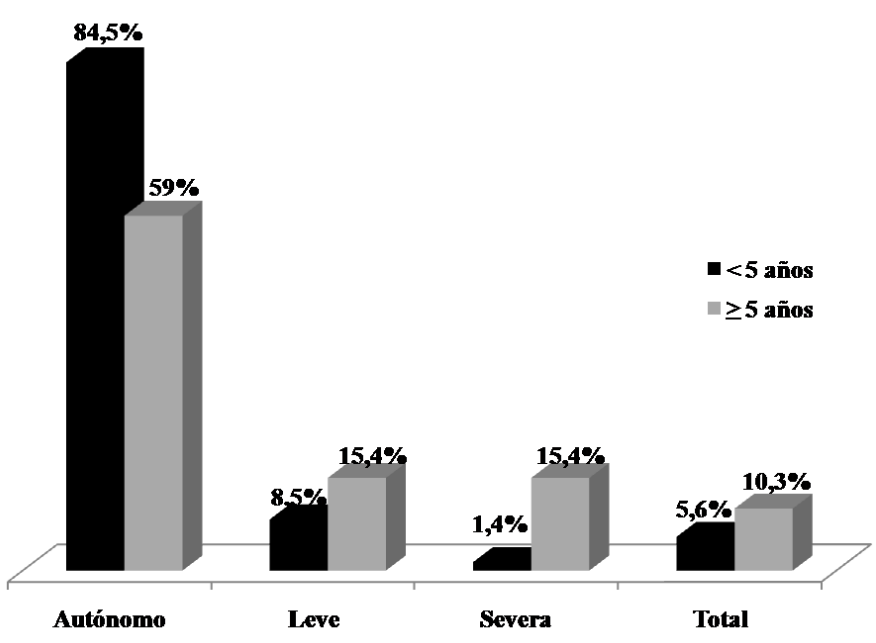

a los retrasos en el diagnóstico incluyendo; falta de conocimiento y experiencia de los profesionales de la salud en EEMM, dificultades para derivar pacientes a centros de referencia, falta de centros especializados o demasiado alejados 0 acceso limitado a servicios de genómica ${ }^{13}$.

En los últimos años se ha implantado en la práctica totalidad de los sistemas autonómicos de salud, los denominados grupos de morbilidad ajustados (GMA) dentro de las estrategias de atención a pacientes con enfermedades crónicas en atención primaria, una herramienta similar a los grupos de riesgo clínicos (CRG) en los que la unidad de análisis no son el evento sino el individuo y que siguiendo una metodología de case-mix, agrupa a los pacientes en categorías según su nivel de riesgo, teniendo en cuenta para ello la presencia de comorbilidades y la complejidad (resultante de valorar el riesgo de ingreso hospitalario, mortalidad, visitas a atención primaria/especializada y gasto en farmacia para cada código diagnóstico $)^{16}$. Los pacientes de nuestra serie presentan a pesar de su corta media de edad, una elevada complejidad asistencial derivada del número de comorbilidades asociadas, las asistencias a consultas con otras especialidades médico-quirúrgicas o en el propio servicio de medicina interna, la necesidad de ingreso hospitalario y el número medio de fármacos por paciente. Esta complejidad, tal y como ha quedado demostrado, guarda relación estrecha también con el retraso en el diagnóstico.

Finalmente, un porcentaje superior al $11 \%$ de nuestros pacientes presenta un nivel de dependencia severa 0 total y cerca de un $10 \%$, un coeficiente intelectual por debajo de 80 en la escala de Wechsler. La discapacidad física 0 intelectual más o menos grave es una característica común a muchas EEMM y comprometen notablemente la calidad de vida de los pacientes. Se da la circunstancia de que los pacientes con mayor grado de discapacidad/ dependencia son precisamente los que han de soportar unos costes más elevados y una peor calidad de vida relacionada con la salud ${ }^{17}$.

Sin duda, el carácter multicéntrico de nuestro estudio ofrece una amplia visión del perfil de pacientes atendidos en las unidades y consultas monográficas de EEMM en los servicios de medicina interna de Galicia. A pesar de la heterogeneidad de las patologías atendidas, el elevado número de variables analizadas, permite concluir fehacientemente el alto índice de complejidad asistencial asociado a este tipo de pacientes así como la importancia de un diagnóstico precoz en su pronóstico clínico y funcional. La inclusión en esta serie de los pacientes atendidos regularmente en una unidad de referencia (CSUR) para enfermedades metabólicas hereditarias puede suponer a priori un sesgo en la selección de los pacientes, sin embargo, los hallazgos encontrados en el cómputo global del estudio son similares a los publicados con anterioridad en series más extensas de pacientes por lo que consideramos una muestra extrapolable a lo que suceda en otros servicios de medicina interna con atención a EEMM. Somos conscientes que muchos pacientes con patologías de baja prevalencia son atendidos regularmente por compañeros internistas al margen de las unidades incluidas en este estudio (consultas de enfermedades autoinmunes, hepatología, etc) y por tanto no están recogidos en nuestra serie, pero creemos que sí están representados en tanto en cuanto, todas las EEMM comparten aspectos comunes que sí han sido analizados en este trabajo.

En definitiva, este es el primer estudio que analiza el perfil clínico de los pacientes con EEMM atendidos en unidades específicas de medicina interna de Galicia. El incremento en la prevalencia de este tipo de enfermedades en los 
últimos años de la mano del mayor conocimiento de las mismas y los programas de cribado, el desarrollo de las técnicas diagnósticas (la biología molecular y diagnóstico genético en particular) y la disponibilidad de nuevas alternativas terapéuticas para algunas de ellas hace prever una mayor volumen de estos pacientes en edad adulta en el futuro inmediato, lo que nos obliga a continuar profundizando en el conocimiento e investigación y asumir cuando menos, la función coordinadora del control de estos pacientes en un entorno multidisciplinar.

\section{Financiación}

La realización del presente estudio no ha sido financiado ni subvencionado por organismo público o entidad privada.

\section{Conflicto de intereses}

Los autores declaran que no tienen ningún conflicto de interés al respecto del presente artículo.

\section{BIBLIOGRAFÍA}

1. Federación Española de Enfermedades Raras [Internet]. [citado 26 de abril de 2020]. Disponible en: https://enfermedades-raras.org/index.php/component/ content/article?id=1318:resumen-informe-de-la-ponencia-del-senado.

2. Datos en cifras sobre Enfermedades Raras I FEDER [Internet]. [citado 27 de abril de 2020]. Disponible en: https://enfermedades-raras.org/index.php/enfermedadesraras/enfermedades-raras-en-cifras.

3. Puente-Ferreras A, Barahona-Gomariz MJ, Fernández-Lozano MP. Las enfermedades raras: naturaleza, características e intervención biopsicosocial. Rare Diseases: Nature, Characteristics and Biopsychosocial Intervention. 2011 11(1):11-23.

4. Gracia D. Las enfermedades raras, un reto histórico. Eidon. 2017 Jun; 47:1-2.

5. J.M. Grau, F. Cardellach. Enfermedades raras y el especialista en Medicina Interna. Med Clin. 2010 Apr:134(12):540-541.

6. Huete García A. Necesidades socio-sanitarias de la población con enfermedades raras que acude a las oficinas de farmacia en España. Pharm Care Espana. 2015 Nov; $17(6): 745-54$

7. Datos en cifras sobre Enfermedades Raras I FEDER [Internet]. [citado 27 de abril de 2020]. Disponible en: https://enfermedades-raras.org/index.php/enfermedadesraras/enfermedades-raras-en-cifras.

8. Cardellach, F. y Ribes, A.(2018). Medicina interna y enfermedades raras. Transición niño-adulto. Arbor. 2018 Jul-Sep; 194 (789): a460

9. INSERM, Orphanet. Portal de información de enfermedades raras y medicamentos huérfanos. [citado 23 de abril de 2019]. Disponible en línea en: https://www.orpha.

10. Linertová R, Valcárcel-Nazco C, Posada-De-la-Paz M, López-Bastida J, SerranoAguilar $P$ and BURQOL-RD Research Network. Disability evaluation in patients with rare diseases in Spain: the importance of being in accord. BURQOL-RD Rare Dis Orphan Drugs 2016, 3 (1): 5-10.

11. García, M., Verde, M. P., Sueiro, J., Rosado, A., \& Sorlí, J. V. Abordando las Enfermedades Raras desde la consulta de Atención Primaria: si se quiere, se puede. Revista Clinica de Medicina de Familia 2013 Feb; 6 (1): 32-36.

12. EURORDIS. The Voice of 12,000 Patients. [citado 27 de abril de 2020]. Disponible en línea en: https://www.eurordis.org/IMG/pdf/voice_12000_patients/ EURORDISCARE_FULLBOOKr.pdf

13. Hazal P-E, Chalandon A-S, Aymé S, Deleuze C. Diagnostic delay in rare diseases: a documented list of (296) rare diseases for which delayed diagnosis would be especially detrimental, based on the French situation. 2020 D0l: 10.21203/ rs.3.rs-32308/v1 (preprint).

14. Yan X, He S, Dong D. Determining How Far an Adult Rare Disease Patient Needs to Travel for a Definitive Diagnosis: A Cross-Sectional Examination of the 2018 National Rare Disease Survey in China. International Journal of Environmental Research and Public Health. 2020 Mar; 17(5).

15. Jo A, Larson S, Carek P, Peabody MR, Peterson LE, Mainous AG. Prevalence and practice for rare diseases in primary care: a national cross-sectional study in the USA. BMJ Open. 2019 Apr;9(4):e027248.

16. González González Al, Miquel Gómez AM, Rodríguez Morales D, Hernández Pascual M, Sánchez Perruca L, Mediavilla Herrera I; Grupo de Trabajo GMA y Grupo de Estratificación del Servicio Madrileño de Salud. Concordancia y utilidad de un sistema de estratificación para la toma de decisiones clínicas [Concordance and usefulness of a stratification system for clinical decision making]. Aten Primaria. 2017 Apr;49(4):240-247.

17. López Bastida, J, Linertová R, Serrano-Aguilar P Hens Pérez M, Posada-De-la-Paz $\mathrm{M}$, Oliva Moreno J. Los costes socioeconómicos y la calidad de vida relacionada con la salud en pacientes con enfermedades raras en España. Revista Española de Discapacidad. 2012 Mar; 1 (1): 251 